\title{
Multiple human-to-human transmission from a severe case of psittacosis, Sweden, January-February 2013
}

\author{
A Wallensten (anders.wallensten@folkhalsomyndigheten.se) ${ }^{1,2}$, H Fredlund 3 , A Runehagen 4 \\ 1. Public Health Agency of Sweden, Solna, Sweden \\ 2. Department of Medical Sciences, Infectious Diseases, Uppsala University, Uppsala, Sweden \\ 3. Department of Laboratory Medicine/ Clinical Microbiology, Communicable Disease Control Unit, Örebro University Hospital, \\ Örebro, Sweden \\ 4. Communicable Diseases Control Unit, Kronoberg County Council, Växjö, Sweden
}

Citation style for this article:

Wallensten A, Fredlund H, Runehagen A. Multiple human-to-human transmission from a severe case of psittacosis, Sweden, January-February 2013 . Euro Surveill. 2014;19(42): pii=20937. Available online: http://www.eurosurveillance.org/ViewArticle.aspx?Articleld=20937

Proven transmission of Chlamydia psittaci between humans has been described on only one occasion previously. We describe an outbreak which occurred in Sweden in early 2013, where the epidemiological and serological investigation suggests that one patient, severely ill with psittacosis after exposure to wild bird droppings, transmitted the disease to ten others: Two family members, one hospital roommate and seven hospital caregivers. Three cases also provided respiratory samples that could be analysed by PCR. All the obtained $C$. psittaci sequences were indistinguishable and clustered within genotype $A$. The finding has implications for the management of severely ill patients with atypical pneumonia, because these patients may be more contagious than was previously thought. In order to prevent nosocomial person-toperson transmission of $C$. psittaci, stricter hygiene measures may need to be applied.

\section{Introduction}

Psittacosis is an infectious disease caused by Chlamydia psittaci, a strict intracellular bacterium. Typical symptoms include abrupt onset of fever, rigors, headache, myalgia, malaise, cough which usually is unproductive and atypical pneumonia [1-2]. Birds are the natural host for the bacterium but other animals including humans can get infected. Humans can get infected after contact with birds by inhaling dried contaminated bird secretions, dried-out droppings or dust from feathers [3]. The incubation period is approximately one to four weeks. Most infected people only experience mild influenza-like disease but severe illness can occur. The disease, which is notifiable by law in Sweden, is not common, with only five to ten cases reported yearly in the years preceding 2013 [4].

Cases are usually sporadic without epidemiological links to a common source. Between January and March 2013, there was an unusual increase in psittacosis cases in southern Sweden, when a total of 17 sporadic cases of psittacosis were reported, distributed across four counties. The annual number of cases in these counties had ranged from one to six during the 10 preceding years. The primary case in this report was one of the sporadic cases. Investigations revealed that the main risk factor for the sporadic cases was exposure to wild birds and their droppings, as previously reported by Rehn et al. The increase in cases was suggested to have been due to weather factors that increased the secretion from affected birds, an unusual epizootic among wild birds, or a more transmissible strain [5].

Person-to-person transmission of has previously not been considered as an important pathway for transmission. It has only been described in two suspected episodes in the literature. In a report from 1977, a patient suffering from pneumonia believed to have been caused by $C$. psittaci transmitted the disease to his son, a neighbour, another patient and to eight hospital staff [6]. However, at the time of this study, the existing serological tests could not discriminate between $C$. psittaci and C. pneumoniae. In light of this and the fact that $C$. pneumoniae is known to spread readily between humans, it is questionable whether the outbreak was caused by $C$. psittaci. This issue has also been discussed by the United States Centers for Disease Control and Prevention [7]. There is, however, one recent documented outbreak with person-to-person transmission of psittacosis [8] that occurred in Scotland in 2012. In the outbreak, the primary case had pneumonia and transmitted the infection to five others. Four of these were family contacts and one a healthcare worker.

\section{Outbreak description}

On 23 January 2013, the communicable disease control unit in Kronoberg County, Sweden, was notified of a patient hospitalised with severe psittacosis. After two weeks, more cases of psittacosis were reported, all with an obvious epidemiological link to the primary case. An investigation was started in order to look into 
the possibility and magnitude of human-to-human transmission.

The primary case, a 73 year-old man was admitted to hospital on 13 January with a three-day history of chills and fever. X-ray imaging showed signs of pneumonia and the patient received intravenous cefotaxime treatment. Despite antibiotic treatment, his condition worsened during the next couple of days. His body temperature rose to above $40^{\circ} \mathrm{C}$ and his oxygen saturation fell from $95 \%$ to $80 \%$. After three days he was transferred to the intensive care unit (ICU) and given moxifloxacin as additional treatment. A bronchoscopy was performed in the ICU and samples from bronchoalveolar lavage was sent for microbiological analysis. Test results came back positive for $C$. psittaci by polymerase chain reaction (PCR) but negative for Legionella pneumoniae, Mycoplasma pneumoniae, influenza virus, respiratory syncytial virus and general bacterial culture. After only one day in ICU he had to be transferred to a university hospital for extracorporeal membrane oxygenation (ECMO) treatment where he was treated for 26 days, after which time he was moved back to the local ICU where he died a month later.

On 25 January, an assistant nurse, who had been taking care of the primary case in the ICU on 18 January, fell ill in what she believed was influenza. After four days with high fever she was admitted to hospital with atypical pneumonia and was diagnosed with psittacosis by PCR.

The same day, 25 January, a doctor who had also worked in the ICU on 18 January fell ill with similar symptoms as the assistant nurse. At that time he was off duty, and suspecting psittacosis, started to treat himself with doxycycline without taking any tests. When he came back to work on 11 February he still had a high C-reactive protein level of $230 \mathrm{mg} / \mathrm{l}$ (normal< $5 \mathrm{mg} / \mathrm{l})$. Initial serological investigation was negative but on repeated sampling he showed evidence of past infection.

The doctor who performed the bronchoscopy also selftreated with antibiotics as soon as he learned of the diagnosis and did not develop any symptoms.

On 28 January an 89 year-old man was diagnosed with pneumonia at the same hospital after falling ill with fever and chills two days earlier. He was admitted and tested positive for psittacosis by PCR. This man had shared a hospital room with the primary case from 14 to 17 January while he was being treated for a cerebral infarction.

On 1 February both the primary case's wife and their son fell ill. Their son lived in the same house as the primary case and his wife. The son showed symptoms compatible with psittacosis, with chills, fever, headache and coughing and received treatment from his local general practitioner. Serological investigation showed evidence of acute infection. The wife of the primary case developed more serious symptoms with high fever and syncope and was admitted to hospital. Her serological test was initially negative but after one month she showed evidence of having had a C. psittaci infection.

Between 28 January and 5 February, five additional staff fell ill on the ward where the primary case was treated before he was transmitted to the ICU. Four were assistant nurses who had been tending to the primary case. The fifth was an assistant nurse who could not remember if she had tended to the primary case, but it is likely that she assisted the nurse responsible for the patient on one occasion. All five at first experienced chills, fever, headache and myalgia. As influenza was circulating at this time, they initially believed they had influenza. However, they were all subsequently diagnosed with pneumonia and three of them were admitted to hospital. When tested, they were negative for influenza, but three showed an acute serological response to $C$. psittaci, one showed signs of infection in follow-up and one was negative in all testing.

\section{Methods}

\section{Epidemiological investigation}

All reported cases of psittacosis in Kronoberg County were interviewed about risk factors and exposure history. Staff working at the hospital were informed of the outbreak.

A confirmed case was defined as a person who had been exposed to the primary case while he was symptomatic and subsequently, within the incubation period for psittacosis, presented with symptoms compatible with a clinical diagnosis of psittacosis, and where no other more likely risk exposures were present. In addition, laboratory confirmation of the diagnosis should have been established. Laboratory confirmation was considered fulfilled if $C$. psittaci was detected in respiratory secretions by PCR, or if a raised IgM antibody titre was detected or an elevation of IgG in two consecutive samples was shown.

A probable case was defined as a person fulfilling the criteria of a confirmed case but lacking other laboratory proof of infection than a C. psittaci IgG titre.

A possible case was defined as a person fulfilling the criteria of a confirmed case but with no laboratory evidence of $C$. psittaci infection.

Incubation periods for the cases were investigated.

\section{Laboratory investigation}

C. psittaci was identified in respiratory samples by amplification of an 84-base pair (bp) fragment of the outer membrane protein A gene (ompA) according to Heddema et al. [9]. The assay was run as a duplex realtime PCR including screening for Legionella species 
Case details, laboratory investigation results and status according to case definition, psittacosis outbreak, Kronoberg County, Sweden, January-February $2013(\mathrm{n}=11)$

\begin{tabular}{|c|c|c|c|c|c|c|}
\hline \multirow{2}{*}{ Case details } & \multirow{2}{*}{$P C R$} & \multicolumn{2}{|c|}{ Serology test 1} & \multicolumn{2}{|c|}{ Serology test 2} & \multirow{2}{*}{ Case status } \\
\hline & & $\lg M$ & $\lg G$ & $\lg M$ & $\lg G$ & \\
\hline Primary case & Positive & Negative & 256 & Not taken & Not taken & Confirmed \\
\hline ICU nurse & Positive & Negative & Negative & Negative & Negative & Confirmed \\
\hline ICU physician ${ }^{\mathrm{a}}$ & Not taken & Negative & Negative & Negative & 64 & Probable \\
\hline Hospital roommate & Positive & Positive & 256 & Negative & 64 & Confirmed \\
\hline Primary case's son & Not taken & Positive & 1024 & Not taken & Not taken & Confirmed \\
\hline Primary case's wife & Not taken & Negative & Negative & Negative & 64 & Probable \\
\hline Ward nurse 1 & Not taken & Negative $^{b}$ & 1028 & Not taken & Not taken & Confirmed \\
\hline Ward nurse 2 & Not taken & Positive & Negative & Positive & Negative & Confirmed \\
\hline Ward nurse 3 & Not taken & Negative & 256 & Not taken & Not taken & Probable \\
\hline Ward nurse 4 & Not taken & Positive & 256 & Not taken & Not taken & Confirmed \\
\hline Ward nurse 5 & Not taken & Negative $^{b}$ & Negative & Negative $^{b}$ & Negative & Possible \\
\hline
\end{tabular}

a Started early antibiotic treatment at first signs of illness.

b Weak reaction, but reported as significant by the laboratory.

and an internal amplification control. In order to determine the genotype of $C$. psittaci, all PCR-positive samples were further investigated by amplification and sequence analysis of a $560 \mathrm{bp}$ fragment of ompA covering variable domain I and II.

IgG and IgM antibodies specific to C. psittaci were shown by microimmunofluorescence performed at a laboratory accredited for this test since the $1990 \mathrm{~s}$ [10]. The serum samples were simultaneously tested for antibodies against $C$. pneumoniae, $C$. trachomatis and $C$. psittaci. Threshold titre for positive test was for IgG $1 / 64$ and for IgM 1/16. Parrot faecal samples were analysed for C. psittaci using the MagAttract Viral RNA M48 extraction kit (Qiagen, Hilden, Germany) and real-time PCR detection of the $23 \mathrm{~S}$ gene, as previously described [11].

\section{Environmental investigation}

The possibility of recovering samples from the primary case's bird feeder was investigated. Faecal samples were taken from an ICU nurse's boyfriend's parrot.

\section{Results}

\section{Epidemiological investigation}

Interviews with the primary case and relatives regarding potential risk factors for psittacosis revealed that the primary case had cleaned a garden bird feeder indoors two weeks before signs of disease. No other connection with domestic or wild birds or their droppings could be identified. He did not live close to a poultry farm or other bird holding. The primary case's son helped to feed the birds when his father was hospitalised. When the father was diagnosed, his son removed the bird feeder and destroyed it by burning.
This took place 12 days before the son himself fell ill. The primary case's wife did not have contact with the birds or the bird feeder.

None of other cases had any history of bird exposure before falling ill except for one of the ICU nurses whose boyfriend had a parrot that she had helped to feed. They did not live close to poultry farms or similar.

In total, in addition to the primary case, six confirmed, three probable and one possible secondary case of psittacosis were identified. Three of these additional cases were male and the median age was 54 years, (range 33-89 years). Case details are summarised in the Table and Figure. Six of the secondary cases were hospitalised. No further transmission from the secondary cases was discovered.

The incubation period ranged from 7 to 20 days in affected cases (mean 12.4) when including all cases. First exposure for the wife and son could not be defined as they had multiple contacts with the primary case.

\section{Laboratory investigation}

The results of the microbiological and serological testing are summarised in the Table. The owner of the parrot was sampled but showed no serological response to psittacosis.

Three cases provided respiratory samples that could be analysed by PCR. All the obtained $C$. psittaci sequences were indistinguishable and clustered within genotype A. 


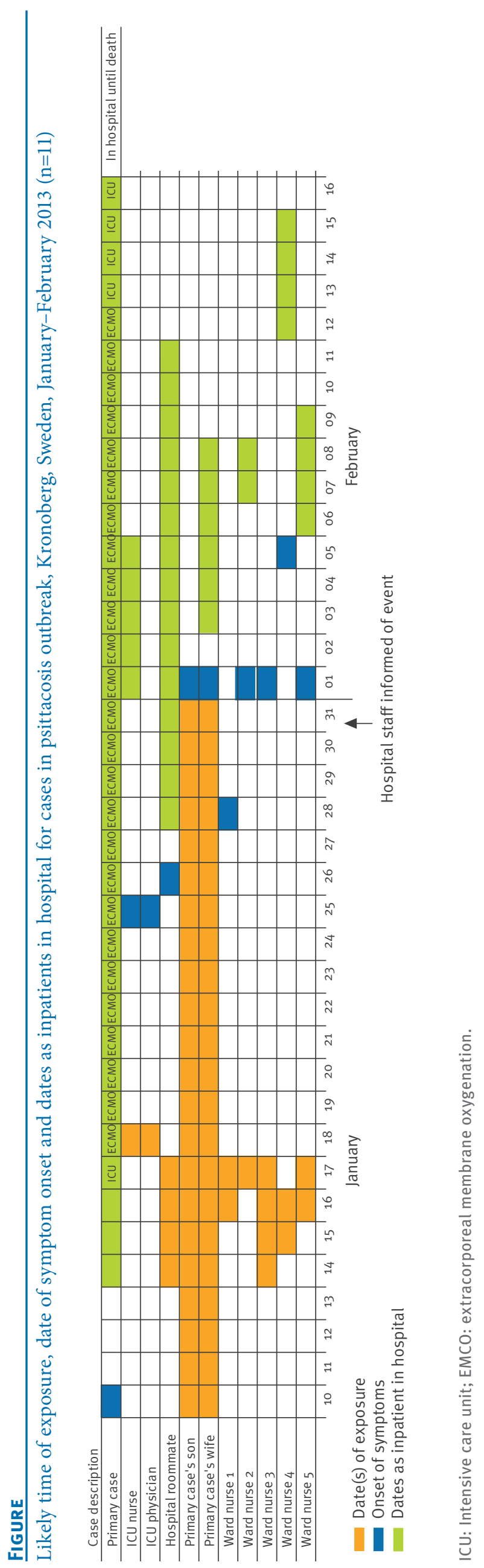

Environmental investigation

Unfortunately no samples could be taken from the bird feeder in the primary case's garden as it had been destroyed. C. psittaci could not be detected in bird droppings from the parrot.

\section{Control measures}

As soon as transmission of the pathogen between patients was suspected, the staff working in the ICU at the time of the incident were informed and asked to seek care should they develop symptoms. The hospital staff on the ward where the patient had initially been treated were informed on 31 January. Instructions were given that all patients with atypical pneumonia should be treated in single ward rooms. The hospital staff were instructed to use filtering face piece (FFP3) masks during procedures with high risk of aerosol-creating procedures such as respiratory training.

\section{Discussion and conclusion}

The primary case in this investigation is likely to have fallen ill from contact with wild birds as one of the many sporadic cases explained by this risk factor at the time [5]. Person-to-person transmission of psittacosis is likely to be rare, but this study clearly supports the previous limited evidence that it may occur. In this outbreak we identified three PCR-confirmed psittacosis cases and seven with less solid evidence of infection, i.e. serological indication only for $C$. psittaci infection. We presume that all these 10 cases were caused by exposure to a primary case with severe disease. All fell ill within the incubation period for the disease after having been exposed to the primary case and no other likely transmission routes could be identified. There is some uncertainty regarding the son of the primary case since he was exposed both to his father during his illness and the bird feeder believed to have been the source of his father's illness. It is therefore impossible to know if he was infected by his father or directly from the bird feeder. The ICU nurse who had a boyfriend with a parrot had most likely been infected by the primary case as the parrot tested negative for the disease and the boyfriend did not have any serological response of psittacosis. Irrespective of the total number, the finding of human-to-human transmission is of significance as it shows that the Scottish incident [8] is not unique and this may have consequences for the management of psittacosis cases.

A few of the cases did not respond with high titres in the serological tests and some only with IgG response. We believe that this may have been because they received early treatment due to high awareness of the disease in the hospital and they were aware that they had been exposed to the primary case. However, we cannot rule out the possibility that those who had only IgG findings may have been the result of past infections. Serological aetiological diagnosis of pneumonia has its limitations. For that reason the patients have been classified into confirmed, probable and possible cases. To set an aetiological diagnosis, both an acute 
phase serum sample and a convalescent serum sample some weeks after the acute infection is often needed unless an IgM test is positive in the acute phase. For that reason the diagnosis may be delayed. Further, cross-reacting antibodies between $C$. psittaci and $C$. pneumoniae have been under discussion [12]. Often a late convalescent serum can be helpful to confirm the aetiology.

As we see it, there are two possibilities for why personto-person transmission took place in this event. The primary case could have been especially contagious or he could have had a $C$. psittaci strain that was especially transmissible. It is well known that strains with the same ompA gene can differ in their virulence [13]. However, as we did not detect onward transmission from the secondary cases and as the limited genetic analysis did not show any abnormalities from other strains, we believe the first theory. In further support of this hypothesis, although it is likely that our primary case and the other sporadic cases in Sweden at the time were infected with the same strain from a wild bird source, there were no reports of onward transmission from the other sporadic cases notified at the time in Sweden. However the contacts of the other cases may not have been followed up as closely. The genotyping of a subset of the sporadic cases believed to have been infected by wild birds between January and March 2013 showed the same type A subtype as our primary and secondary cases who were positive by PCR. All had genotype $A$, which is mainly associated with parrots and other psittacine birds but which has also been found in passerine birds [14]. It is the genotype causing most human cases worldwide [15]. But to completely rule out the possibility of a more pathogenic strain being the reason for the increased transmissibility in this outbreak, whole genome analysis is required. This could not be performed due to lack of an isolate. We believe that the primary case was more contagious because he was very ill and therefore excreted more bacteria. In support of our theory of increased risk of transmission from severely ill patients, the data on incubation periods for infected cases shows a possible dose response association. Those who were highly exposed, like the nurse and the doctor who treated the primary case in the ICU, and the patient sharing a room with the case, had a shorter incubation time $(7,7$ and 10 days respectively) than the cases who were only exposed to the patient while caring for him on the ward and who had an average of 15 days before symptoms started (range 11-20 days) (Figure). However, due to the low number of cases in the outbreak, more observational studies like this one are needed to show whether this is correct. Although the ICU nurse who attended at the bronchoscopy fell ill, we believe that the shorter incubation period had more to do with the patient having become more severely ill and thus being treated in ICU than the bronchoscopy procedure itself, as the doctor who fell ill was not present at the procedure and only examined the patient.
It seems probable that our preventative measures did not prevent any further transmission since all of the secondary cases were related to the primary case. He had already been transmitted to ECMO-treatment in a university hospital when the staff were informed and stricter hygienic measures regarding treatment of patients with atypical pneumonia were implemented. It is likely, however, that the measures may have shortened the duration of illness of some of the secondary cases as they are likely to have received treatment earlier than they would otherwise have done.

\section{Public health implications}

Our previous report of the unusual increase of psittacosis in Sweden this year concluded that psittacosis is likely to be a more common disease in Sweden than previously thought, as our study suggested that it may be overlooked by clinicians and not tested for in cases of atypical pneumonia by laboratories unless specifically requested. The fact that we have now shown that nosocomial transmission may occur from seriously ill patients increases the importance of diagnosing cases of atypical pneumonia correctly, as it has implications for the management of patients with pneumonia. In order to prevent nosocomial transmission from patients with psittacosis, enhanced protection may be needed when caring for severely ill patients with atypical pneumonia, for example, using airway protection with facemasks and treating the cases in isolation. Staff and others exposed to a psittacosis patient should also be informed of the symptoms so that they seek care should they fall ill.

Authors' contributions

A Wallensten drafted, finalised and submitted the manuscript. H Fredlund was responsible for the serological laboratory investigation during the outbreak and for writing the laboratory investigation part of the manuscript which he also helped revise. A Runehagen managed the outbreak, contributed with all epidemiological information regarding the outbreak and helped to draft and revise the manuscript.

\section{References}

1. Stewardson AJ, Grayson ML. Psittacosis. Infectious disease clinics of North America. 2010;24(1):7-25. http://dx.doi. org/10.1016/j.idc.2009.10.003

2. AP, Grayson ML. Psittacosis--a review of 135 cases. The Medical journal of Australia. 1988;148(5):228-33.

3. Smith KA, Bradley KK, Stobierski MG, Tengelsen LA, National Association of State Public Health Veterinarians Psittacosis Compendium C. Compendium of measures to control Chlamydophila psittaci (formerly Chlamydia psittaci) infection among humans (psittacosis) and pet birds, 2005. J Am Vet Med Assoc. 2005;226(4):532-9. http://dx.doi.org/10.2460/ javma.2005.226.532

4. Smittskyddsinstitutet. Statistik för papegojsjuka 2013 [Accessed 21 Oct 2014]. Swedish. Available from: http://www.folkhalsomyndigheten.se/amnesomraden/ statistik-och-undersokningar/sjukdomsstatistik/ papegojsjuka-/

5. Rehn M, Ringberg H, Runehagen A, Herrmann B, Olsen $B$, Petersson AC, et al. Unusual increase of psittacosis in southern Sweden linked to wild bird exposure, January to April 2013. Euro Surveill. 2013;18(19):20478. 
6. Broholm KA, Bottiger $M$, Jernelius H, Johansson $M$, Grandien $M$, Solver K. Ornithosis as a nosocomial infection. Scand J Infect Dis. 1977;9(4):263-7.

7. Compendium of measures to control Chlamydia psittaci infection among humans (psittacosis) and pet birds (avian chlamydiosis), 1998. Center for Disease Control and Prevention. MMWR Recomm Rep. 1998;47(RR-10):1-14.

8. McGuigan CC, Mclntyre PG, Templeton K. Psittacosis outbreak in Tayside, Scotland, December 2011 to February 2012. Euro Surveill. 2012;17(22): $\mathrm{pii}=20186$

9. Heddema ER, Beld MG, de Wever B, Langerak AA, Pannekoek Y, Duim B. Development of an internally controlled real-time PCR assay for detection of Chlamydophila psittaci in the LightCycler 2.0 system. Clin Microbiol Infect. 2006;12(6):571-5. http:// dx.doi.org/10.1111/j.1469-0691.2006.01417.x

10. Gnarpe J, Naas J, Lundback A. Comparison of a new commercial EIA kit and the microimmunofluorescence technique for the determination of IgG and IgA antibodies to Chlamydia pneumoniae. APMIS. 2000;108(12):819-24. http://dx.doi. org/10.1111/j.1600-0463.2000.tbooo04.X

11. Ehricht R, Slickers P, Goellner S, Hotzel H, Sachse K. Optimized DNA microarray assay allows detection and genotyping of single PCR-amplifiable target copies. Mol Cell Probes.

2006;20(1):60-3. http://dx.doi.org/10.1016/j.mcp.2005.09.003

12. Stralin K, Fredlund H, Olcen P. Labsystems enzyme immunoassay for Chlamydia pneumoniae also detects Chlamydia psittaci infections. J Clin Microbiol. 2001;39(9):3425-6. http://dx.doi.org/10.1128/ JCM.39.9.3425-3426.2001

13. Miyairi I, Laxton JD, Wang X, Obert CA, Arva Tatireddigari VR, van Rooijen N, et al. Chlamydia psittaci genetic variants differ in virulence by modulation of host immunity. J Infect Dis. 2011;204(4):654-63. http://dx.doi.org/10.1093/infdis/jir333

14. Olsen B, Persson K, Broholm KA. PCR detection of Chlamydia psittaci in faecal samples from passerine birds in Sweden. Epidemiol Infect. 1998;121(2):481-4. http://dx.doi.org/10.1017/ So950268898001320

15. Harkinezhad T, Geens T, Vanrompay D. Chlamydophila psittaci infections in birds: a review with emphasis on zoonotic consequences. Vet Microbiol. 2009;135(1-2):68-77. http:// dx.doi.org/10.1016/j.vetmic.2008.09.046 